\title{
The Role of Atmospheric Conditions in Determining Intensity of Crepuscular and Anticrepuscular Rays
}

\author{
Matthew S. Van Den Broeke, William H. Beasley, and Michael B. Richman \\ School of Meteorology, University of Oklahoma, Norman, Oklahoma
}

(Manuscript received 21 July 2009, in final form 28 January 2010)

\begin{abstract}
During the summer of 2006, daily observations of crepuscular and anticrepuscular rays were made around sunset from central Oklahoma. A relative scale of ray intensity was developed and used to relate the rays to concurrent meteorological conditions. Evidence is presented suggesting that both topography and clouds can cast shadows leading to rays. Relationships are hypothesized between estimated ray intensity and atmospheric variables, and predictive equations are developed for ray intensity and assessed using data from the summer of 2008. Except for a few cases in which rays were traced back to topography east of the Rocky Mountains, the most intense rays were produced by towering cumulus $(\mathrm{Cu})$ and cumulonimbus $(\mathrm{Cb})$ clouds to the west after hot days with light winds.
\end{abstract}

\section{Introduction and motivation}

\section{a. Description of crepuscular and} anticrepuscular rays

Crepuscular rays (CRs) appear as alternating dark and light bands across the sky and can be observed at any time of day. They are common, often taking the form of light streaks as sunlight filters through cloud gaps. In this paper, the focus will be on CRs occurring when the sun is on or below the horizon. At these times, they typically take the form of dark stripes extending upward from where the sun has set. Anticrepuscular rays (ARs) are virtually identical, except they converge on the antisolar point, which, when the sun is just below the western horizon, is just above the eastern horizon (Albright 1937). If ARs are visible, they correspond to specific CRs, which may also be visible. In rare cases, rays extend across the entire sky.

There is not much information about these phenomena in the literature. A description of CRs was provided by Minneart in his classic work on optics. He described them as "radiating from the imaginary point below the horizon where the sun is" and described ARs as formed in a similar way, except observed at sunset "against the purple background of the counter-twilight where they converge

Corresponding author address: Matthew S. Van Den Broeke, School of Meteorology, 120 David L. Boren St., Norman, OK 73072.

E-mail: matthew.vandenbroeke@ou.edu towards the anti-solar point" (Minneart 1954). Minneart also explained how ARs and CRs correspond exactly, though from the ground, the near-horizon sections are seen most readily. The ARs have been described as "rare and evanescent" (Monteith 1990). Nevertheless, we have observed them frequently from central Oklahoma.

Lynch (1987) describes the optical theory of CR visibility, saying they are most prevalent when the sun is near or below the horizon and "are most common when the air is clear." Observations from Oklahoma suggest CRs may be observed when the sky is cloudless or hazy, but are most common under nearly cloud-free conditions. Next, Lynch notes the rays are most visible within $15^{\circ}$ of the sun and the antisolar point (i.e., near each horizon). We found this to be largely true in central Oklahoma, though full-sky displays were not extremely rare. Ray visibility, except for a few exceptional events, was maximized near each horizon. According to Lynch, ARs are most intense very near the antisolar point and are usually associated with large clouds overhead or near the eastern horizon and are seldom observed from a small cloud, unless the cloud is near the antisolar point (Lynch 1987). In our observations, however, ARs that were not traceable to any visible clouds frequently occurred-thus, clouds or topographic features over the horizon may also be responsible. Finally, Lynch notes that maximum ray color contrast with the background sky should occur near the antisolar point, while maximum ray intensity contrast should occur near the sun. Our observations agreed; ARs 
typically stood out as dark blue stripes across a pinkish horizon, while CRs were nearly the same color as the background sky and visible because of their relative dimness.

\section{b. Optics of crepuscular and anticrepuscular rays}

Crepuscular and anticrepuscular rays are visible because their brightness contrasts with that of the surrounding sky. Even if shadows are cast into the atmosphere, rays will not be seen without this contrast, which results from scattering. Assuming a homogenous spatial distribution of scattering particles and particle scattering efficiencies, contrast is essentially a function of the difference between light scattering inside versus outside the shadow (Lynch 1980).

Lynch (1987) explains common observations of crepuscular and anticrepuscular rays. Rays are more frequent when the sun is near the horizon and appear most intense near the horizon because these conditions are associated with the greatest pathlength through the umbras (densest part of cloud shadows), increasing contrast with the sky outside the ray. Rays are also most frequent and intense when atmospheric visibility is relatively high because contrast is reduced when aerosol optical thickness is high. Smaller clouds have relatively short umbras, which should not as easily reach across the sky to the antisolar point, though in Oklahoma ARs were often observed with no visible clouds. We therefore hypothesize that distant topography and deep, optically thick clouds are the sources of many shadows seen as rays in Oklahoma. Maximum intensity contrast occurs with CRs because the western sky is closer to the shadow's source, and less cumulative diffusion of light into the umbra allows it to be darker than farther east. Light within the rays comes from scattering of sky light, possibly by clouds (Lynch 1987).

\section{c. Ray formation mechanisms}

Rays may become visible whenever a dark shadow is cast by an object, such as a terrain feature, deep cloud, or dense smoke plume. In the first known published reference to CRs, Heim (1916) notes in passing that clouds and mountains can cause them. Douglass (1916) notes the appearance of many well-defined CRs and ARs in the twilight sky over Arizona and attributes them to cloud shadows. One must wonder, however, if some of the rays he observed were caused by topography. Minneart discusses only cloud shadows as a cause of the rays, and develops an equation allowing one to find distance to the causative cloud (Minneart 1954). For a cloud three miles above the surface, Minneart shows that rays could be observed 450 miles from the cloud. In Oklahoma, many CRs and ARs can be attributed to clouds, mainly cumulus and cumulonimbus. Well-developed cumulonimbus can be much taller than three miles, so rays from them could theoretically be visible beyond 450 miles. When considering how far from a shadow-casting object CRs will be visible, though, it is necessary to keep in mind that only certain atmospheric conditions and aerosol content will render them visible.

Topographically generated shadows were first discussed at length by Lynch (1980). Though he does not specifically cite mountains as a cause of CRs and ARs, one can infer that an observer well removed from a mountain might see its shadow as a ray under appropriate conditions. This is especially true because mountains are tall, like deep convective clouds, and individual summits could cast individual shadows, much like cumuliform cloud towers. The formation of CRs and ARs by mountains was described by Monteith (1986), who attributes a series of CRs visible near Hyderabad, India, to the Western Ghats, a coastal mountain range some $475 \mathrm{~km}$ (300 mi) west of the observation site. The geography of this region, including the Western Ghats sloping down to the Deccan Plateau on which Hyderabad is located, is similar to the geographical situation in Oklahoma, where the terrain slopes gradually from the Rocky Mountains toward the Gulf of Mexico. Furthermore, Williams (1987) attributes CRs observed from an island in the Aegean Sea to a mountain range in southern Yugoslavia, about 260 miles $(420 \mathrm{~km}$ ) distant.

\section{d. Atmospheric conditions leading to ray visibility}

To the best of our knowledge, no previous research has examined the relationship between occurrence of CRs or ARs and meteorological variables, though several papers have described some necessary atmospheric conditions, mostly in terms of airborne dust and aerosols.

In his treatment of CR formation by mountains, Monteith (1986) discusses required particle properties for $\mathrm{CR}$ and $\mathrm{AR}$ formation. Soil dryness, vegetation, and smoke contribute to atmospheric particle content over India, which is also true over Oklahoma. In particular, during the dry summer of 2006, many airborne particles could likely be attributed to dry soil and smoke. During the summer of 2007, however, which was one of the wettest summers recorded in central Oklahoma, spectacular displays of CRs and ARs were also frequently observed.

Monteith (1986) notes that scatterer density is important to ray visibility. Enough particles must be present so sufficient scattering can produce visible ray contrast, but too many scatterers may cause light to become overly scattered, causing loss of contrast. This suggests an optimal optical thickness. In India, Monteith observed rays to be most distinct just after the monsoon ended, presumably when the fewest large particles were airborne. Ray visibility decreased thereafter until eventually the rays could no longer be seen-presumably scatterer density had become too high. According to Monteith (1986), 
TABLE 1. Categories used to estimate ray intensity.

\begin{tabular}{cc}
\hline & Scale used to estimate ray intensity \\
\hline Ray intensity & Description \\
\hline 0 & No rays visible \\
1 & Rays barely visible with detailed scrutiny \\
2 & Rays visible with detailed scrutiny, but \\
& not when quickly glancing at the sky \\
3 & Rays barely visible when quickly glancing \\
& at the sky \\
4 & Rays moderately visible when quickly \\
& glancing at the sky \\
5 & Rays extremely well defined; obvious \\
& with a quick glance at the sky \\
\hline
\end{tabular}

ARs become visible when particles have a diameter comparable to the wavelength of the light being scattered. Sky-wide ray displays occur when scattering is independent of direction, evidence that the particles responsible are smaller than the wavelength of light. In Oklahoma, clay particles and forest fire smoke are the most likely sources of particles within this size range (Schaefer and Day 1981).

Predictability of these ray phenomena is currently low. Monteith later returned to India and found that CRs formed by the Western Ghats had disappeared. Thus, he raised an unanswered question: why do rays sometimes appear frequently, even for many evenings in a row, and then disappear for years when viewed from the same location (Monteith 1990)?

\section{e. Goals of this study}

In this study, we attempt to identify meteorological and aerosol conditions under which CRs and ARs are most likely visible. It is shown that rays observed from central Oklahoma are likely caused by both clouds and topographic features. Relationships are examined between CR and AR intensity and meteorological variables. Finally, predictive equations for ray intensity are developed from the summer of 2006 observations, and data from the summer of 2008 are used to verify their usefulness. While these results were specifically derived over central Oklahoma during summer, we speculate they may be generalized for much of the Great Plains where meteorological processes and topography are similar. Application may also be possible to other areas of the world where rays are sometimes visible.

\section{Methodology}

During the summer of 2006, the Rays At Sunset Over the Plains Experiment (RASOPEX) was carried out in Norman, Oklahoma. Intensities of CRs and ARs were recorded subjectively on each noncloudy evening according

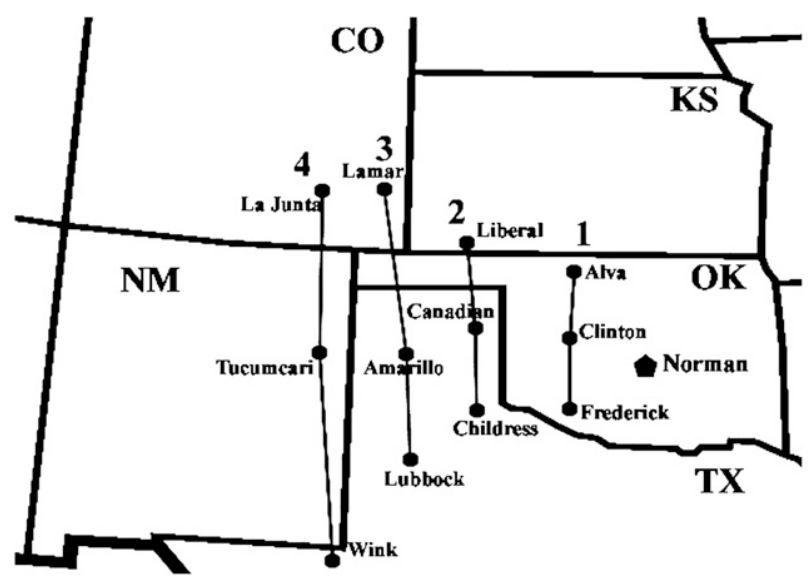

FIG. 1. The four north-south transects used in this study. Numbers correspond to the transect numbers described in the text. Labeled cities denote the locations of sensors from which meteorological data were obtained.

to the scale described in Table 1 . If ray intensity varied on a given evening, the highest observed value was recorded. Wind speed and rainfall data were collected from Norman and for four approximately north-south-oriented transects to the west, shown in Fig. 1. In addition, regional visible and infrared satellite imagery was gathered each evening when ray intensity was observed.

For days when CR and AR intensity was observed, archived Automated Surface Observing Station (ASOS) data were collected. Meteorological variables gathered included wind speed at 1200, 1500, and $1800 \mathrm{CDT}$, and 1800 CDT temperature, dewpoint, relative humidity, and sea level pressure. These data were analyzed using S_PLUS 8.0, a statistical analysis program (available online at http://spotfire.tibco.com/products/s-plus/ statistical-analysis-software.aspx), and transect averages were calculated for all variables by averaging the three values on a given transect. These wind, temperature, and moisture variables were chosen to estimate subjectively the amount of windblown dust and to indicate synoptic conditions locally and along transects to the west. Though not analyzed, relative humidity aloft may also be an important variable since it is strongly related to aerosol characteristics.

To assess the impact of aerosols on ray appearance, archived aerosol data were gathered. These included the mass concentration of particles with diameter $<2.5 \mu \mathrm{m}$ (PM2.5) and aerosol optical depth (AOD) at $550 \mathrm{~nm}$, a measure of aerosol quantity in an atmospheric column. PM2.5 data were collected at the surface and represent the average hourly concentration between 1500 and 2200 UTC. AOD data were collected by the satellites Terra and Aqua. These satellites collect the same data, so an average of the two values was taken. For all aerosol 
TABLE 2. Categories for most common cloud type at sunset, and their assigned codes.

\begin{tabular}{cccc}
\hline \hline \multicolumn{4}{c}{ Codes for dominant cloud type } \\
\hline Code & Dominant cloud type & Code & Dominant cloud type \\
\hline 0 & None & 3 & Shallow cumulus \\
1 & Stratus & 4 & Midheight cumulus \\
2 & Cirrus & 5 & Cumulonimbus \\
\hline
\end{tabular}

data, the value at $35^{\circ} \mathrm{N}, 99^{\circ} \mathrm{W}$ was used, determined by interpolation and estimation. A discussion of potential error sources in AOD measurement is provided in Kondragunta et al. (2008).

Several variables were derived from satellite imagery. A $60^{\circ}$ sector was defined on each evening of observation, centered in the direction of the sun at sunset and extending westward to the fourth transect. Two satellite variables were approximate percentage of this sector obscured by visible and infrared cloud cover, estimated to the nearest $10 \%$. Another variable was defined as the distance to the nearest visible or infrared cloud cover from Norman within this sector, to the nearest $5 \mathrm{~km}$. If clouds were nearly overhead, a value of $5 \mathrm{~km}$ was recorded. A fourth satellite variable was the minimum infrared temperature observed within the sector to the nearest $5^{\circ} \mathrm{C}$. In most cases, coldest temperatures were associated with cumuliform clouds. Finally, the most common cloud type within the sector was assessed from satellite imagery and given a numerical value as described in Table 2. While scatterplots of ray intensity versus cloud type provide useful information, cloud types were assigned numerical values in order of expected importance for ray formation in order to include cloud type as a meaningful variable in the predictive equations. It was expected that stratus and cirrus would be less likely to cast shadows visible as rays than any cumuliform clouds, and that deep cumuliform clouds would more likely produce rays than shallow cumuliform clouds.

Finally, archived 0000 UTC soundings were examined from Norman, Oklahoma (KOUN); Amarillo, Texas (KAMA); and Dodge City, Kansas (KDDC) for each evening on which CR and AR intensity were observed. Sounding variables gathered included convective available potential energy (CAPE; in $\mathrm{J} \mathrm{kg}^{-1}$ ), convective inhibition (CIN; in $\mathrm{J} \mathrm{kg}^{-1}$ ), equilibrium level (EL; in $\mathrm{hPa}$ ), lifting condensation level (LCL; in m), LCL-level wind speed (in kt), level of free convection ( $\mathrm{LFC}$; in $\mathrm{hPa}$ ), and 500 -hPa lifted index $\left(\mathrm{LI}\right.$; in $\left.{ }^{\circ} \mathrm{C}\right)$. Additional sounding variables included Norman (local) 850-hPa geopotential height and temperature, 24-h change in these quantities, 500-hPa geopotential height and temperature, 24-h change in $500 \mathrm{hPa}$ geopotential height, and 300-hPa wind speed. These variables were chosen to represent the synoptic situation and to assess stability.
A rectangular 90-variable predictor matrix was constructed for 38 days of CR observations and 34 days of AR observations. Scatterplots were created of observed $\mathrm{CR}$ and $\mathrm{AR}$ intensity versus each predictor variable, and Pearson's correlation (hereafter referred to as correlation) was calculated for each variable pair. Stepwise regression was used to isolate approximately 20 best-correlated predictor variables for each ray type. Correlation was computed between each pair of these variables, and for each pair with correlation $\geq 0.5$, at least one member was removed to ensure robust predictive equations. Multiple regression was carried out using the remaining variables, and the adjusted $r$-squared method was used to choose optimal predictive equations. Comparable meteorological data and ray intensity observations from the summer of 2008 were used to assess the performance of the predictive equations.

\section{Results}

\section{a. Overview of observations}

Observations of CRs and ARs were attempted from 16 May to 7 July 2006, except for one day. Rays were not produced on 10 overcast days ( $23.5 \%$ of days). In the 39 remaining days, no rays were visible on 8 days $(20.5 \%)$ and some rays were visible on 31 days $(79.5 \%)$. The average intensity of 34 AR observations was 2.24; the average intensity of $38 \mathrm{CR}$ observations was 1.89 . Rays were observed more frequently than suggested by the literature, perhaps indicating exceptionally favorable atmospheric and/or geographical conditions, or pointing to a paucity of deliberate observations. ARs were observed slightly more frequently than CRs, on 26 (76.5\%) versus 24 of possible days, despite the fact that ARs are thought of as rare phenomena (e.g., Monteith 1990).

\section{b. Case studies}

A few case studies are presented to show the role of both clouds and topography in ray formation. The first occurred on the evening of 27 April 2006. A large area of thunderstorms was located over the Oklahoma and northwest Texas Panhandles, southwest Kansas, and southeast Colorado (Fig. 2a), though in Norman the sky was cloudless. About 8-12 dim CRs were observed across the northwestern sky. Over about $15 \mathrm{~s}$, a single CR suddenly became very well defined and then dimmed. Since significant topography to the west was obscured by thunderstorms, clouds likely caused the rays. No ARs were observed on this occasion, though ARs traceable to clouds have been observed.

Topography was also a source of ray formation. On 4 and 5 June, a ray appeared across the southern sky in the same position at the same time each evening and slowly 

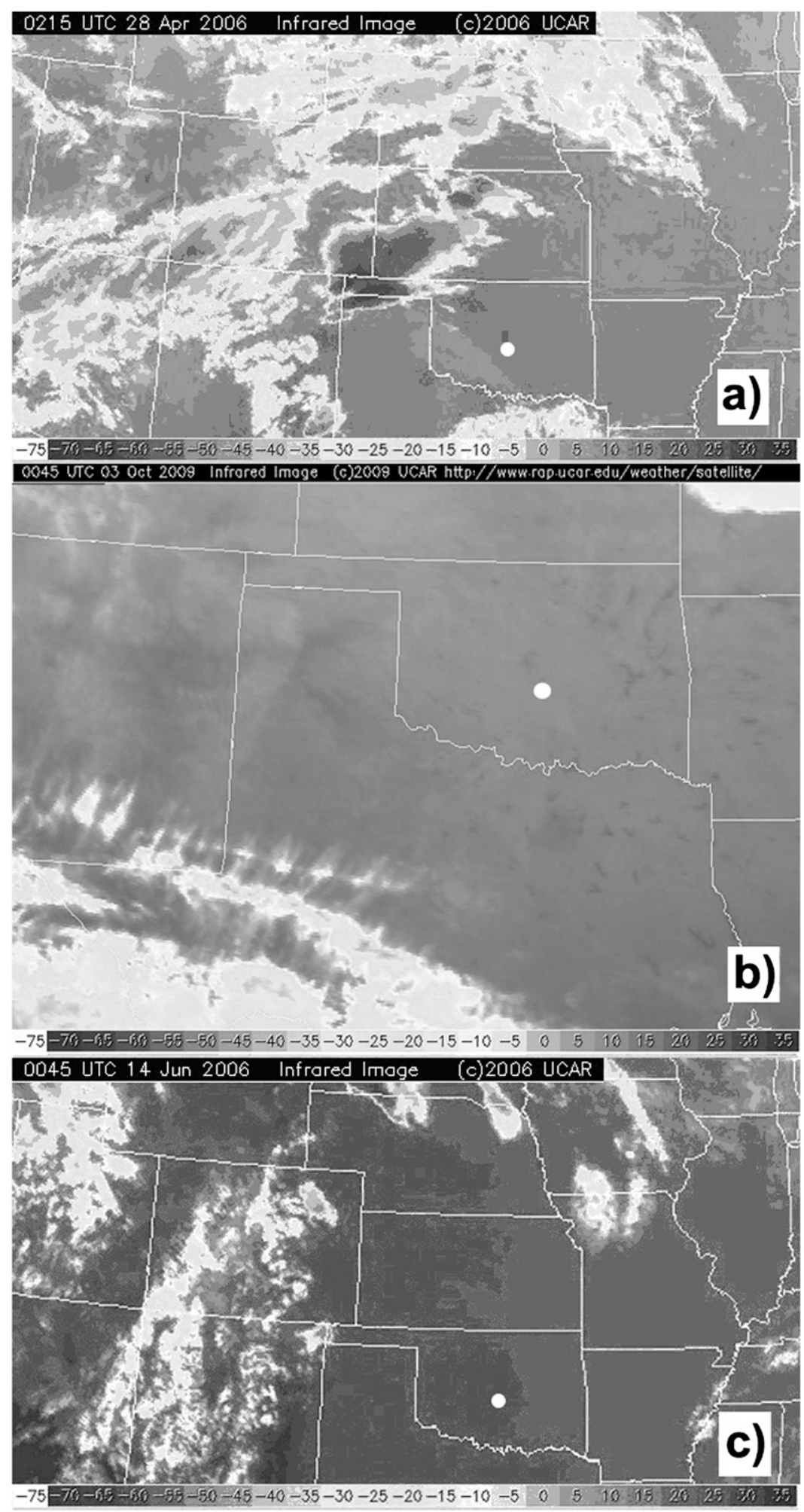

FIG. 2. Infrared satellite imagery from (a) 0215 UTC 28 Apr 2006, (b) 0045 UTC2 Oct 2009, and (c) 0045 UTC 14 Jun 2006. White circles indicate the observation location (Norman, OK). 
moved northward while weakening. The same ray pattern was visible on numerous other evenings through June. Cloud patterns were different on some of these evenings, so the similar appearance and behavior of this ray from evening to evening is attributed to topography. The same ray pattern and behavior was again observed later in the summer, and through the summers of 2007-09. A similar phenomenon was observed on the evening of 2 October 2009, when no clouds were present between Norman and the Rocky Mountains (Fig. 2b). In this case, the time of ray disappearance corresponded to sunset approximately 260 miles $(420 \mathrm{~km})$ west of Norman, suggesting a terrain feature somewhere near the Texas-New Mexico border may have been responsible. In some cases, the Rocky Mountains in Colorado or New Mexico, approximately 320 miles $(515 \mathrm{~km})$ distant, likely cast shadows visible as rays from central Oklahoma.

A spectacular ray display on 13 June 2006 seems to be attributable to both topography and clouds. On this evening, small cumulus and one thunderstorm were present to the west of Oklahoma along the mountains (Fig. 2c). About 15 min after local sunset, four very dark rays appeared in both east and west, one of which appeared to be the topographic ray described above. Throughout the display, smaller rays were noted to split off the major CRs and move northward. By the end of the display, any small cumulus east of the Rocky Mountains would have been in the mountains' shadows.

Rays were sometimes observed before sunrise. On 22 September 2006, striking CRs and ARs were observed 15-20 min before sunrise. From Norman, the sky appeared cloudless to the east. Satellite imagery showed deep convective clouds over Arkansas, which were likely responsible for the rays.

\section{c. Correlation between ray intensity and predictor variables}

We acknowledge that many of the correlation coefficients reported here are small. The best predictor variables may have not yet been identified, or the natural variability and complexity of these phenomena may preclude correlation of higher magnitude between ray intensity and any one meteorological variable. If ray events caused by clouds could be isolated from those caused by topographic features, predictability of ray intensity should be higher. Visibility of topographic shadows should be most related to aerosol concentration, while cloud-generated shadows should be more sensitive to meteorological variables.

\section{1) Crepuscular RAys: Important VARiables}

A correlation value of -0.366 was found between CR intensity and 1200 CDT wind speed in Norman (Fig. 3a).
Though there were many exceptions, strongest 1200 CDT wind speeds were typically associated with the weakest CRs, while lighter winds were associated with more intense rays. If too much dust is present, multiple scattering should decrease contrast between shadowed and unshadowed regions of sky (Monteith 1986). Sufficient particles for scattering are likely always present over Oklahoma, so even with light winds CRs may be visible. A similar association was also noted on transects to the west-if a shadow encounters any region of higher dust concentration, it will be weakened, potentially to invisibility for points to the east.

Correlation between CR strength and 1800 CDT Norman temperature was near zero (0.072). Strong rays (strength $=4$ or 5 ), however, were found to occur with warmer temperatures (generally $\geq 85^{\circ} \mathrm{F}$, Fig. $3 \mathrm{~b}$ ). This was also true for all four transects: no strong rays occurred when transect 3 temperature was less than $85^{\circ} \mathrm{F}$, while no strong rays occurred for transect 4 temperature below $90^{\circ} \mathrm{F}$. High temperatures often occur with light winds, since on the southern plains during summer, subtropical high pressure often produces both conditions simultaneously (Chang and Smith 2001). Thus, the presence of subtropical high pressure may indicate favorable conditions for CR visibility.

A correlation of 0.341 was found between CR strength and transect 2 dewpoint at 1800 CDT (Fig. 3c). Low average dewpoint along transects 2 and 3 was associated with weak rays - though few such cases exist, those with average dewpoint $<32^{\circ} \mathrm{F}$ had very weak or no rays (ray strength $=0$ or 1 ). During the spring and early summer, dry air on a western transect might indicate a dryline and cumulus field to the east, leading to rays visible from central Oklahoma. During summer, however, most convection on the southern plains is free convection, and in this case cumuliform clouds are more likely to develop with higher low-level dewpoints. The highest correlation was found with transects in the Texas Panhandle, suggesting an optimal range to cumuliform clouds of about ' 200 miles (320 km) for resultant cloud shadows to be visible from Norman. Satellite observations support these results-CR visibility was associated with the dominant cloud type (Fig. 3d). Ray intensity is here divided into no rays (intensity $=0$ ), weak rays (intensity $=1$ or 2 ), and intense rays (intensity $=3,4$, or 5). Events with cloud types 0, 1, and 2, respectively, representing no clouds, stratus, and cirrus, were associated with no or weak CRs; only one case of intense rays (intensity $=3$ ) was observed when these were dominant. Intense rays were associated with cumuliform clouds (categories 3, 4, or 5) except in one case, though weak rays also occurred with all categories of cumuliform clouds.

No rays occurred when minimum IR temperature was $>-20^{\circ} \mathrm{C}$ (corresponding to an average cloud-top height 
CRS vs. Noon Wind (Norman)

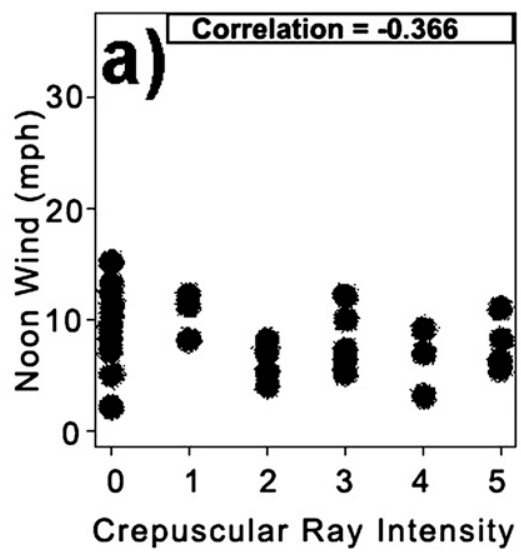

CRS vs. Tr. 26 PM Dewpoint

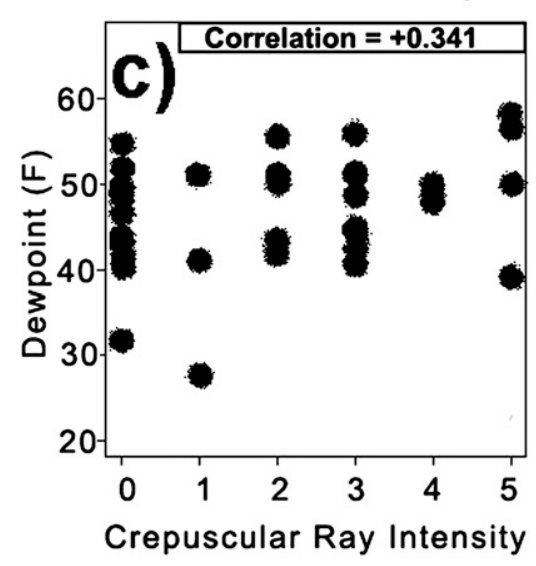

CRS vs. 6 PM Temp. (Norman)

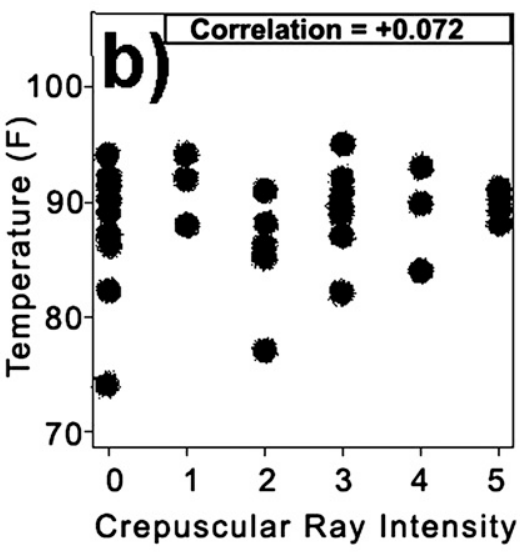

CRS vs. Dominant Cloud Type

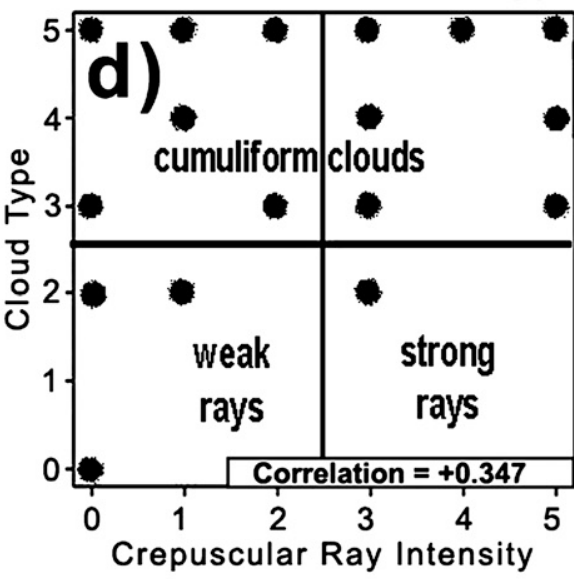

CRS vs. CIN (KOUN)

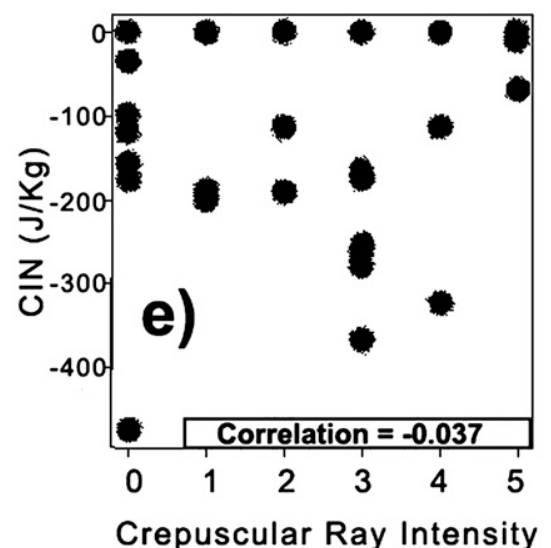

FIG. 3. Crepuscular ray strength vs atmospheric, sounding, and satellite-derived variables: (a) 1200 CDT wind speed in Norman, (b) 1800 CDT temperature in Norman, (c) average 1800 CDT dewpoint on transect 2, (d) dominant satellite-inferred cloud type, and (e) 0000 UTC KOUN CIN. 
ARS vs. 6 PM Temp. (Tr. 2)

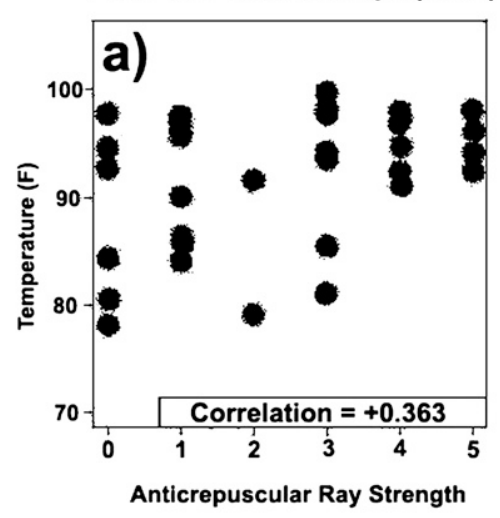

ARS vs. LCL Hght (KOUN)

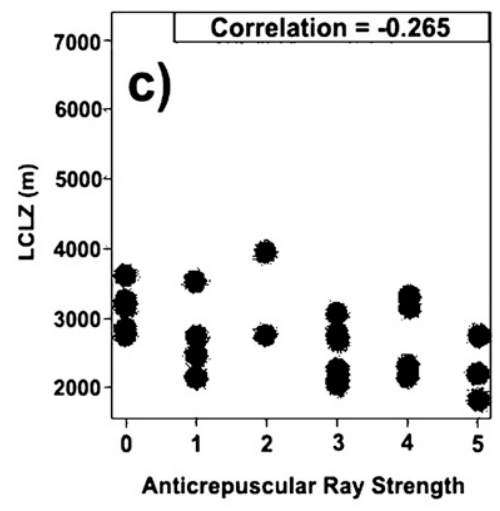

ARS vs. CAPE (KOUN)

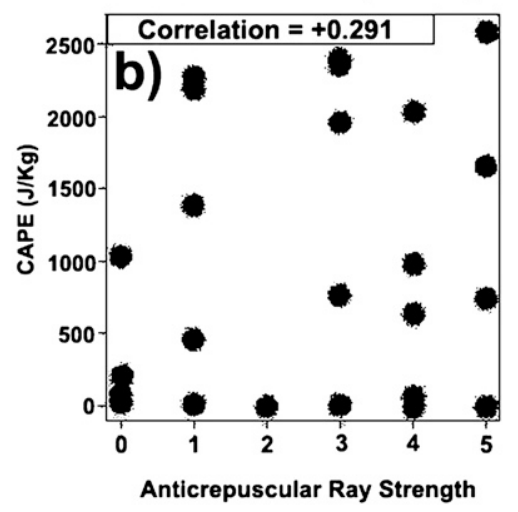

ARS vs. Dominant Cloud Type

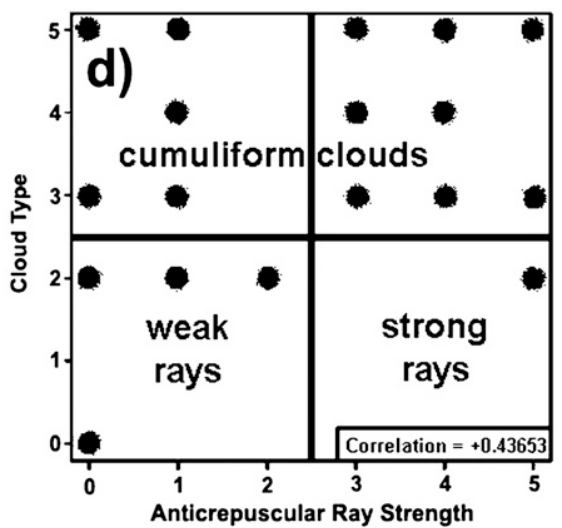

FIG. 4. Anticrepuscular ray strength vs atmospheric, sounding, and satellite-derived variables: (a) average 1800 CDT temperature on transect 2, (b) 0000 UTC KOUN CAPE, (c) 0000 UTC KOUN LCL height, and (d) dominant satellite-inferred cloud type.

of approximately $8.1 \mathrm{~km}$ ), while all cases of intense CRs (ray intensity $=4$ or 5 ) had minimum IR temperature $\leq-40^{\circ} \mathrm{C}$ (corresponding to a cloud-top height of about $10.6 \mathrm{~km})$. Thus, rays caused by clouds required cloudtop height exceeding $8.1 \mathrm{~km}$, and intense rays required cloud-top height exceeding $10.6 \mathrm{~km}$.

Most cases of intense CRs (ray strength $=3,4$, or 5) occurred when CIN was $\leq-250 \mathrm{~J} \mathrm{~kg}^{-1}$ at KOUN (Fig. 3e); a similar threshold applied to KDDC soundings found moderate to strong rays (intensity $=2,3$, or 4 ) in all cases. Substantial CIN may restrict updrafts to a small fraction of the sky, allowing contrast between sunlit and shaded regions. Days with substantial CIN are favored under subtropical high conditions, when subsidence often produces an inversion aloft.

\section{2) ANTICREPUSCULAR RAYS: IMPORTANT VARIABLES}

Fewer variables were found to be well correlated with AR intensity. The literature suggests ARs should be favored when clouds are present near the observer (Lynch 1987). Though this is not necessarily true for central Oklahoma, it is hypothesized that ARs are more reliant on clouds nearer the observer than those involved in the formation of CRs.

On transect 2, the coolest temperatures were associated with weak-to-moderate ARs (intensity $=0,1,2$, or 3 ) while intense rays (intensity $=4$ or 5 ) always occurred when transect average temperature exceeded $90^{\circ} \mathrm{F}$ (Fig. 4a). For higher temperatures, higher CAPE might also be expected. When no ARs were observed, CAPE values exceeding $1000 \mathrm{~J} \mathrm{~kg}^{-1}$ were rare, while rays were always observed for CAPE exceeding $1200 \mathrm{~J} \mathrm{~kg}^{-1}$ (Fig. 4b). The highest CAPE case was also associated with the most intense AR category, but overall, CAPE did not appear to have high predictive value. High local CAPE may promote the development of deep cumulus if clouds can initiate and their updrafts reach the LFC. There may also be some minimum CAPE value above which cumuliform clouds are more common and rays are more likely visible. Substantial CIN 

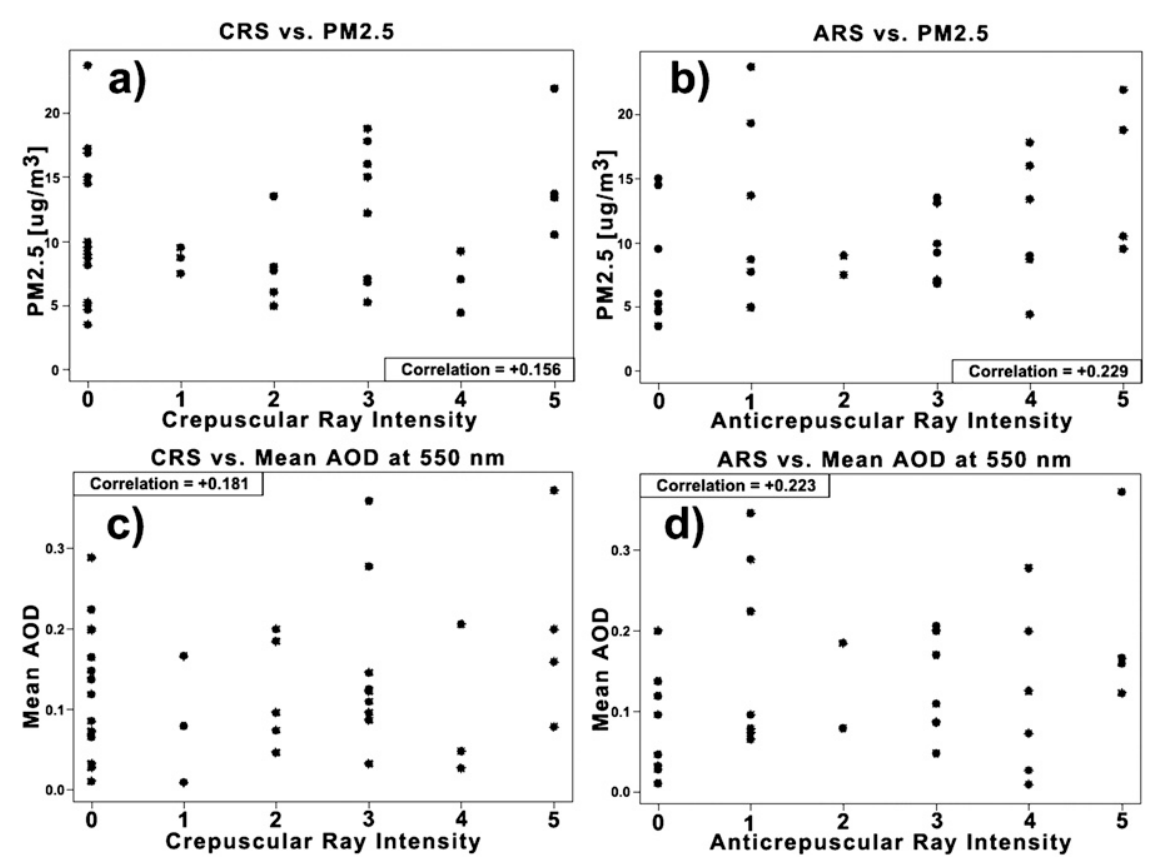

FIG. 5. Scatterplots of (left) crepuscular and (right) anticrepuscular ray intensity vs aerosol concentrations. (a),(b) Ray intensity vs PM2.5 and (c),(d) ray intensity vs mean AOD at $550 \mathrm{~nm}$.

was often present on days with nontrivial CAPE, so in these cases having more CAPE may not improve the potential for deep cumuliform clouds and consequent rays.

Cases without visible ARs were associated with local LCL of $\geq 2800 \mathrm{~m}$, while cases with the most intense ARs had an LCL of $\leq 2800 \mathrm{~m}$. While correlation is again relatively small ( -0.265 ; Fig. $4 c)$, lower LCL appears to favor the most intense ARs. A lower LCL should allow clouds to form more easily and perhaps for updrafts to reach the LFC more readily. With more clouds nearby, ARs would more likely be visible.

As with CRs, days on which cloud type was dominated by stratus, cirrus, or on which there were no clouds were characterized by weak rays (Fig. 4d). All intense AR events except one occurred with cumuliform clouds (cloud type $=3,4$, or 5); weak rays were also observed to occur with all categories of cumuliform clouds. Though any cloud may cast a shadow, cumuliform clouds have greater values and contrasts of optical thickness.

\section{3) RELATIONSHIPS WITH AEROSOL CONCENTRATION}

Relationships between aerosol content and ray intensity were weak. PM2.5 concentration was weakly correlated with CR intensity (0.156; Fig. 5a) and AR intensity (0.229; Fig. 5b). Correlation with AOD at $550 \mathrm{~nm}$ was similarly small for both CRs (0.181; Fig. 5c) and ARs (0.223; Fig. $5 \mathrm{~d})$. There was no apparent decrease in ray intensity at higher aerosol concentration. A majority of the most intense ray events occurred with an AOD of $>0.11$ and PM2.5 concentration of $\geq 9 \mu \mathrm{g} \mathrm{m}^{-3}$.

Aerosol variables should help explain variations in ray intensity. A complication of using these variables, however, is the slant path of the shadows. Instead of using point or column aerosol measurements, best results would be obtained by accounting for variable aerosol content along the length of a shadow. This would require a careful geometrical analysis of each ray. In addition, an improved optical model would need to be developed in the vein of Lynch's theory (1987). Given these improvements, stronger correlations may be found between aerosol content and ray intensity.

\section{4) LAG CORRELATION}

Lag correlation was calculated for each ray type to assess repeatability of ray intensity from one evening to the next. For CRs, 26 pairs of successive nights were available (Fig. 6a). Correlation is 0.445 for these data-thus, about $20 \%$ of evening-to-evening variance in CR intensity can be accounted for by the preceding evening's observation. For ARs, only 19 data pairs were available (Fig. 6b). The ARs appear less similar from evening to evening (correlation $=0.159$ ), as expected given the possibly more local nature of ARs. For both CR and AR intensity, the equations developed below have better predictive ability than the preceding evening's observation. 

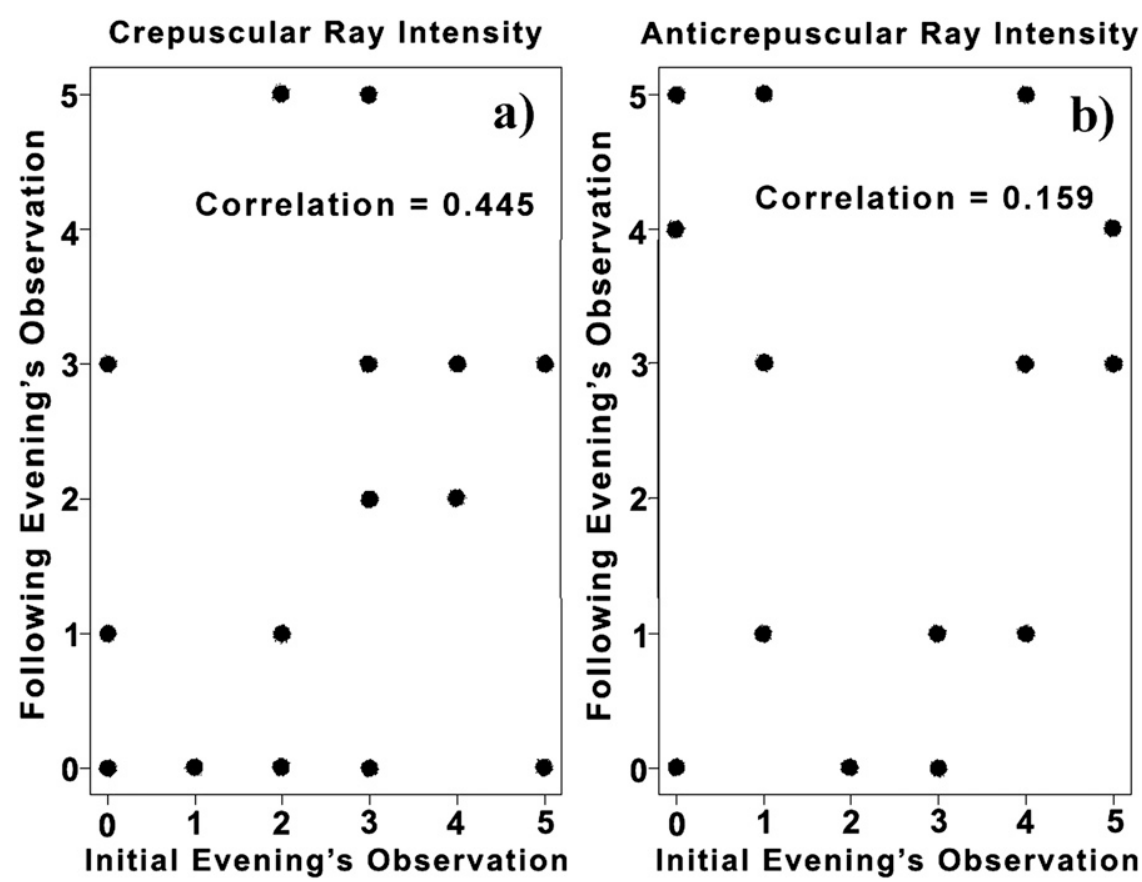

FIG. 6. Lag correlation for (a) crepuscular ray strength and (b) anticrepuscular ray strength.

\section{d. Predictive equations for ray intensity}

\section{1) Crepuscular Rays}

The adjusted $r$-squared method identified the prediction equation using four predictors as optimal; it explains $28.0 \%$ of variance in ray intensity:

$$
\begin{aligned}
\mathrm{CRI}= & -0.5279-0.1552(a)-0.0373(b) \\
& +0.0629(c)+0.2741(d),
\end{aligned}
$$

where CRI $=$ crepuscular ray intensity, $a=1200 \mathrm{CDT}$ (Norman) wind speed (in $\mathrm{mph}$ ), $b=$ average daily rainfall on transect 3 (in hundredths of an in.), $c=$ average 1800 CDT dewpoint on transect $2\left({ }^{\circ} \mathrm{F}\right)$, and $d=$ dominant cloud type to the west (as described in Table 2).

These statistically chosen predictor variables support the prior discussion of favorable conditions for $\mathrm{CR}$ visibility. A variable representing local wind speed was selected, since stronger winds loft more scatterers than are favorable for CR visibility. The CRs were predicted to become less visible as rainfall increased on transect 3 . This was a surprising result, since rainfall to the west could indicate clouds, which would cast shadows visible as rays. Also, rainfall may remove large particles from the air, making rays more visible. Rainfall recorded on a western transect was often associated with extensive thunderstorms and anvil cover. Thus, rainfall here may indicate cloud cover too extensive for contrasts visible as rays. Higher dewpoint on transect 2 correlated positively with ray intensity. All else equal, higher dewpoint should allow clouds to develop more readily. As expected, more intense rays were predicted to occur with cumuliform clouds, and deeper cumuliform clouds were predicted to produce rays of greater intensity.

\section{2) ANTICREPUSCULAR RAYS}

The adjusted $r$-squared method identified the prediction equation using three predictors as optimal; it explains $28.6 \%$ of variance in ray intensity:

$\mathrm{ARI}=-5.1932+0.0717(a)-0.0428(b)+0.4499(c)$,

where: ARI $=$ anticrepuscular ray intensity, $a=$ average $1800 \mathrm{CDT}$ temperature on transect 4 (in ${ }^{\circ} \mathrm{F}$ ), $b=\mathrm{LCL}-$ level wind speed from the 0000 UTC KDDC sounding (in $\mathrm{kt}$ ), and $c=$ dominant cloud type to the west (as described in Table 2).

Locally measured variables were not very predictive of AR intensity-rather, a set of variables representing distant conditions was selected. As average 1800 CDT temperature on transect 4 increased, ARs became more visible. Also, low LCL-level wind speed at KDDC was predicted to yield more intense rays. Both suggest calm weather on the high plains west of the observation site, and are typically realized under subtropical high pressure. Cumuliform clouds, especially those of greater vertical extent, were predicted to yield more intense ARs. This makes sense, since the formation mechanism of 

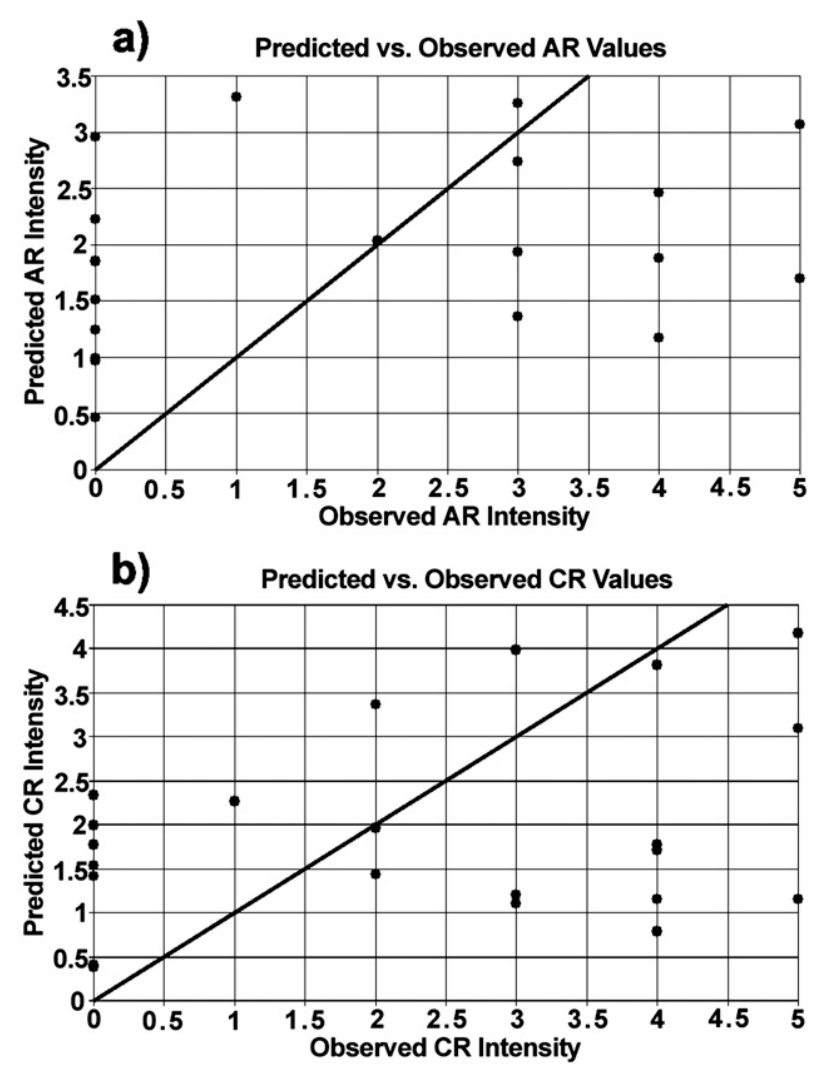

FIG. 7. Predicted vs observed ray intensity values for (a) anticrepuscular rays and (b) crepuscular rays. The black line in each scatterplot indicates a perfect prediction.

many ARs is likely clouds to the west. Deep cumuliform clouds have the greatest optical thickness and cast shadows most likely to remain visible to the eastern horizon.

\section{e. Verification of the predictive equations}

Ray observations were carried out on 22 clear evenings from 18 May to 5 August 2008. On each evening, the intensity of CRs and ARs was assessed, and all necessary observations were collected to evaluate the predictive equations. Although an Alaskan volcano began erupting in mid-July, ray observations were kept until 5 August, which is justified by AOD measurements. Mean intensity of 22 CR (AR) observations was 2.32 (1.91).

Correlation between predicted and true ray intensity values for the verification dataset was 0.320 for ARs $\left(r^{2} \sim 0.102\right)$ and 0.326 for CRs $\left(r^{2} \sim 0.106\right.$; Fig. 7$)$. The predictive equation for CRs worked best when predicted ray intensity was $>3$ (Fig. 7b). While correlation between predicted and observed ray intensity did decrease as expected for the test dataset, the presence of moderate correlation suggests the models capture some relevant physical processes. Discrepancies could be due to the different synoptic regime between the summers of 2006 and 2008 and the presence of volcanic aerosols near the end of the testing dataset. On the southern high plains, the summer of 2006 was dominated by a ridge aloft and characterized by many clear, hot days with convection near the Rocky Mountains or over the Texas and Oklahoma Panhandles. In contrast, the summer of 2008 was characterized by frequent troughs in the upper-level flow and consequent incursions of cooler air (Fig. 8). Eruptions of Okmok Volcano began on 12 July 2008, and subsequent ray observations may have been minimally affected by scattering off volcanic aerosols rather than being dominantly a result of synoptic conditions.

\section{Conclusions}

The CRs were favored when winds were light through the preceding afternoon, indicating that too many airborne particles increase optical thickness so much that contrasts between sunlit and shaded regions are reduced. Warmer 1800 CDT temperature was also found to yield stronger rays, as were locally weak LCL-level wind and moderate CIN. These conditions are typical of those experienced when subtropical high pressure is located over the southern plains. Cumuliform clouds to the west favored CR visibility; as did a higher dewpoint on transect 2 . The resulting prediction equation for $\mathrm{CR}$ strength explained approximately $28 \%$ of variance in ray intensity. ARs were favored when $1800 \mathrm{CDT}$ temperature and dewpoint were high, and when local CAPE $\geq$ $1200 \mathrm{~J} \mathrm{~kg}^{-1}$ was present. These factors suggest local instability should be present for ARs to be highly visible. As for CRs, cumuliform clouds were associated with stronger ARs. The predictive equation for ARs indicated intense rays when conditions were consistent with calm weather along the transect nearest the Rocky Mountains, and when cumuliform clouds were most common to the west. It explained approximately $29 \%$ of variance in ray intensity. Predictive equations were better at forecasting ray intensity than the previous evening's observations. Without accounting for the slant path of shadows, measures of aerosol quantity were poorly correlated with ray intensity.

Summer of 2008 data was used to verify the predictive equations. For each ray type, predicted versus observed intensity had a correlation around 0.32 . Though not as good as for the training dataset, this moderate correlation indicates that the prediction equations capture the essential atmospheric processes responsible for creating intense crepuscular and anticrepuscular rays when the sun is near the horizon. 

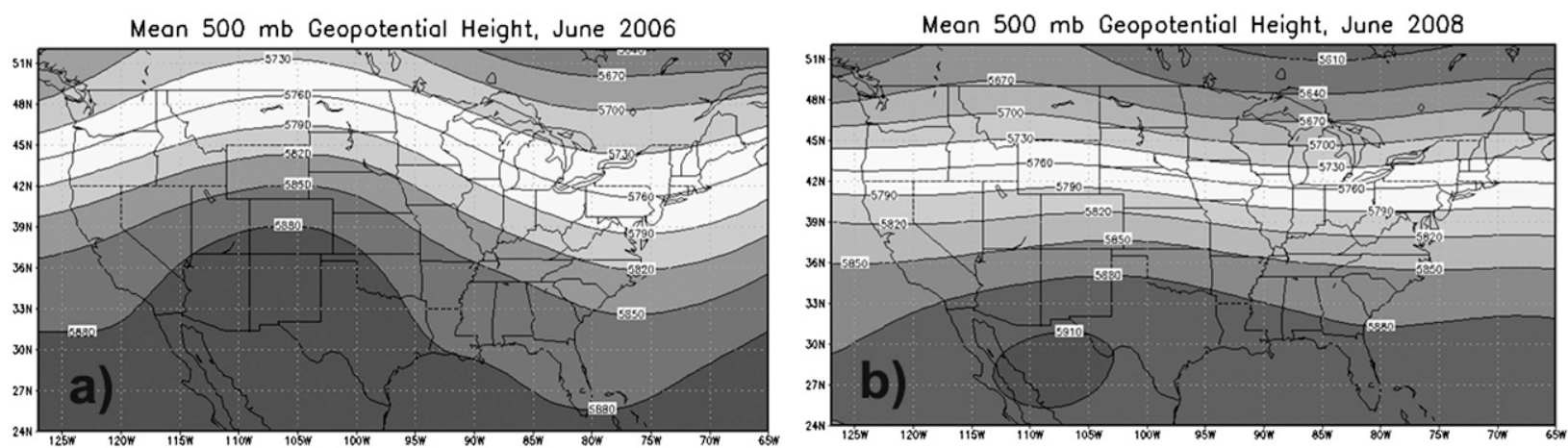

Mean $700 \mathrm{mb}$ Geopotential Height, June 2006
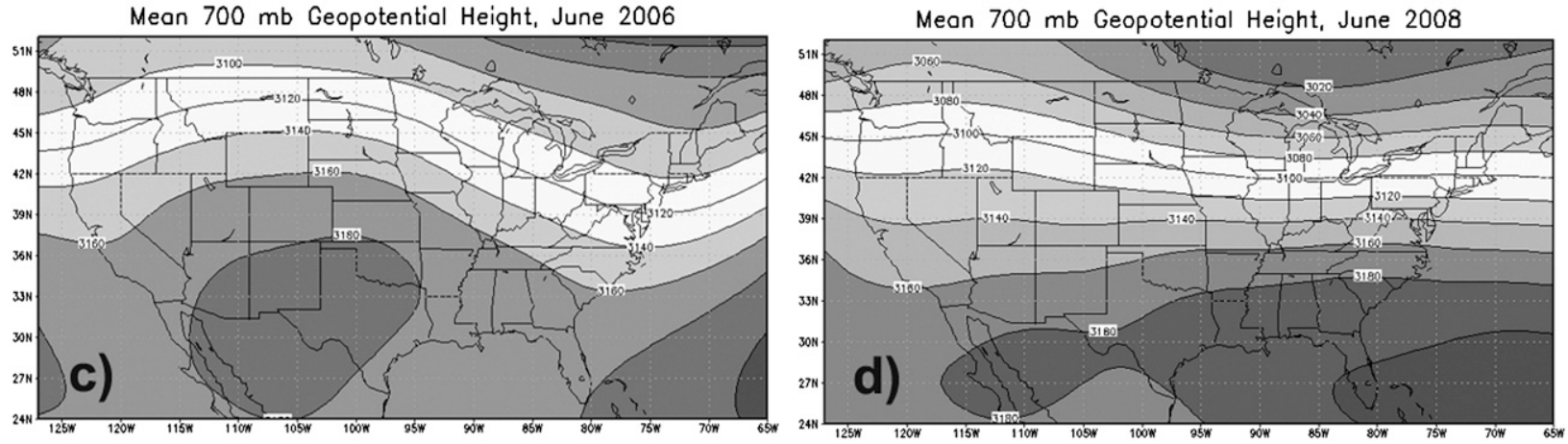

FIG. 8. Mean June synoptic conditions during (left) 2006 and (right) 2008. (a),(b) Mean 500-hPa geopotential height and (c),(d) mean 700-hPa geopotential height.

Acknowledgments. The authors thank two anonymous reviewers who provided many constructive suggestions through the revision process. This paper is substantially improved because of their help. This work was primarily supported by NSF Grants ATM-0646892 and ATM-0733539.

\section{REFERENCES}

Albright, J. G., 1937: An observation of anticrepuscular rays. Mon. Wea. Rev., 65, 3.

Chang, F., and E. A. Smith, 2001: Hydrological and dynamical characteristics of summertime droughts over U.S. Great Plains. J. Climate, 14, 2296-2316.

Douglass, A. E., 1916: Twilight phenomena in Arizona, September to December, 1916. Mon. Wea. Rev., 44, 625-626.
Heim, A., 1916: Explanations of the western purple light and the eastern afterglow (nachgühen). Mon. Wea. Rev., 44, 624-625.

Kondragunta, S., and Coauthors, 2008: Air quality forecast verification using satellite data. J. Appl. Meteor. Climatol., 47, $425-442$.

Lynch, D. K., 1980: Mountain shadow phenomenon. 2: The spike seen by an off-summit observer. Appl. Opt., 19, 1585-1589.

- 1987: Optics of sunbeams. J. Opt. Soc. Amer., 4A, 609-611.

Minneart, M., 1954: The Nature of Light and Colour in the Open Air. Dover, 362 pp.

Monteith, J. L., 1986: Crepuscular rays formed by the Western Ghats. Weather, 41, 292-299.

_ 1990: Crepuscular rays: Reversibility and rarity. Weather, $\mathbf{4 5}$, 187-188.

Schaefer, V. J., and J. A. Day, 1981: A Field Guide to the Atmosphere. Houghton Mifflin, 359 pp.

Williams, M. C., 1987: Crepuscular rays observed in Greece. Weather, 42, 197-198. 